\title{
Neoadjuvant chemotherapy combined with limb salvage surgery in patients with limb osteosarcoma of Enneking stage II: a retrospective study
}

This article was published in the following Dove Press journal:

OncoTargets and Therapy

26 May 2017

Number of times this article has been viewed

\author{
Guangke Yuan ${ }^{1,2}$ \\ Jingming $\mathrm{Chen}^{2}$ \\ Dongjin $\mathrm{Wu}^{\prime}$ \\ Chunzheng Gao' \\ 'Department of Orthopedics, \\ The Second Hospital of Shandong \\ University, Jinan, ${ }^{2}$ Department of \\ Orthopedics, Yidu Central Hospital \\ of Weifang, Weifang, Shandong, \\ People's Republic of China
}

Objective: To explore the effect of neoadjuvant chemotherapy combined with limb salvage surgery in patients with limb osteosarcoma of Enneking stage II.

Patients and methods: Medical records of 98 patients who met the inclusion criteria were retrospectively analyzed. Of these patients, 56 cases who received neoadjuvant chemotherapy combined with limb salvage surgery were listed as group A, while another 42 patients who received limb salvage surgery combined with adjuvant chemotherapy were listed as group B. The recurrence and metastasis rate, survival rate, limb function and incidence of adverse reactions were compared between the two groups.

Results: All 98 patients completed the treatment in this study. Baseline characteristics showed no significant differences between group A and group B, including age, gender, tumor location, maximum tumor diameter and Enneking stage (all $P>0.05$ ). The total metastasis and recurrence rate of group A was significantly lower than that of group B $(25.0 \%$ vs $47.6 \%$, $\left.\chi^{2}=5.419, P=0.020\right)$. The Kaplan-Meier method showed that progression-free survival (PFS) (log-rank $\left.\chi^{2}=4.014, P=0.045\right)$ and overall survival (OS) (log-rank $\left.\chi^{2}=3.859, P=0.049\right)$ of group A were both significantly higher than those of group $\mathrm{B}$. There was no significant difference in the incidence of grades III-IV adverse reactions between the two groups (all $P>0.05$ ). The excellent and good rate of limb function in group A was significantly higher than that in group B $\left(83.9 \%\right.$ vs $\left.66.7 \%, \chi^{2}=3.982, P=0.046\right)$.

Conclusion: Neoadjuvant chemotherapy combined with limb salvage surgery for patients with Enneking stage IIA or IIB limb osteosarcoma patients has better efficacy and can significantly improve limb function of patients.

Keywords: limb osteosarcoma, neoadjuvant chemotherapy, limb salvage surgery, adjuvant chemotherapy

\section{Introduction}

Osteosarcoma is the most common orthopedic malignancy and often occurs in distal femur, proximal humerus, proximal tibia and metaphysic. ${ }^{1}$ The age with peak incidence rate ranges from 14 to 26 years old. ${ }^{2}$ The main clinical symptoms of osteosarcoma include progressive local pain, lumps, swelling, dysfunction and occasionally visible pathological fracture. The oncologic outcomes of osteosarcoma are poor because of its high malignancy and characteristics prone to metastasis. ${ }^{3}$ The goal of osteosarcoma treatment is to control the primary tumor and (micro-)metastatic deposits, to maintain maximal extremity function and to minimalize the treatment-related adverse reactions. ${ }^{4}$

With the improvement of requirements to quality of life, limb salvage surgery has become the main way of treatment of limb osteosarcoma with satisfying local
Correspondence: Chunzheng Gao Department of Orthopedics, The Second Hospital of Shandong University, No 247 North Park Street, Tianqiao District, Jinan 250100, Shandong Province,

People's Republic of China

Tel +86 I5I 53169697

Email prfgao@sina.com 
control and short-term efficacy and yet limited long-term efficacy. ${ }^{5}$ The inclusion of aggressive polychemotherapy into an interdisciplinary treatment concept has led to dramatic prognostic improvements in young patients with seemingly localized extremity disease, with 5-year overall survival rates of $\sim 50 \%-80 \%$ reported by specialized centers or multicentric groups. ${ }^{6}$ Neoadjuvant chemotherapy with its great advantages has been widely used in the treatment of osteosarcoma. First, neoadjuvant chemotherapy can reduce the boundaries of osteosarcoma, which contributes to the implementation of radical resection of the tumor and limb retention; second, neoadjuvant chemotherapy can eliminate the potential micrometastases and prolong relapse-free survival period. ${ }^{7}$ Therefore, neoadjuvant chemotherapy combined with limb salvage surgery for the treatment of non-metastatic osteosarcoma has been widely accepted by physicians and patients. Kudawara et $\mathrm{al}^{8}$ demonstrated the excellent outcome of neoadjuvant chemotherapy combined with limb salvage surgery for patients with limb osteosarcoma. A study by Bacci et $\mathrm{al}^{9}$ also indicated that neoadjuvant chemotherapy combined with limb salvage procedures did not compromise the outcome of patients with limb osteosarcoma. However, there are few studies or reports evaluating the feasibility of neoadjuvant chemotherapy combined with limb salvage surgery for Chinese patients with limb osteosarcoma. Therefore, we designed this retrospective study aiming to compare short-term efficacy, treatment-related adverse reactions and long-term survival between neoadjuvant chemotherapy combined with limb salvage surgery, and limb salvage surgery combined with adjuvant chemotherapy, for patients in Shandong province, China.

\section{Patients and methods}

\section{Patients}

This study was approved by the ethics committee of the Second Affiliated Hospital of Shandong University. When admitted, all the guardians of enrolled patients signed informed consent that the patients' medical records could be used for clinical research in the future. Inclusion criteria are as follows: 1) a histological diagnosis of limb osteosarcoma by biopsy; 2) no distant metastasis confirmed by computed tomography (CT) scan and radionuclide bone scan; 3) Enneking stage IIA or IIB; 4) the main nerve, blood vessels are not involved; 5) patients without congenital disease, cardiovascular disease and respiratory disease; and 6) patients without chemotherapy contraindications and anesthetic allergy.

From January 2010 to December 2013, a total of 98 patients met the inclusion criteria. Of these patients, 56 cases received neoadjuvant chemotherapy combined with limb salvage surgery and were listed as group A, while another 42 patients received limb salvage surgery combined with adjuvant chemotherapy and were listed as group B.

\section{Drugs}

The chemotherapy drugs used in this study were as follows: epirubicin (EPI; Pfizer Pharmaceuticals Ltd., Jiangsu, China; H20000496); cisplatin (DDP; Biological Valley Pharmaceutical Co., Ltd., Yunnan, China; H20043888); methotrexate (MTX; Yue Kang Pharmaceutical Group Co., Ltd., Beijing, China; H20113120); ifosfamide (IFO; Pfizer Pharmaceuticals Ltd.; H10950292); calcium folinate (CF; Hengrui Medicine Co., Ltd, Jiangsu, China; H20010615) and mesna (Pfizer Pharmaceuticals Ltd.; H10950290).

\section{Treatment}

Patients in group A received neoadjuvant chemotherapy combined with limb salvage surgery. Neoadjuvant chemotherapy regimen was EPI + DDP + MTX + IFO: DDP $\left(95 \mathrm{mg} / \mathrm{m}^{2}\right)$, $\mathrm{d} 1,+$ EPI $\left(85 \mathrm{mg} / \mathrm{m}^{2}\right), \mathrm{d} 1-3,+$ MTX $\left(10 \mathrm{~g} / \mathrm{m}^{2}\right), \mathrm{d} 4-10,+$ IFO $\left(2 \mathrm{~g} / \mathrm{m}^{2}\right), \mathrm{d} 15-19$. Adequate hydration and diuretics were prescribed after injection of DDP. After the first injection of MTX, CF $\left(15 \mathrm{~g} / \mathrm{m}^{2}\right)$ was administered once every 6 hours 14 times. In order to prevent cystitis, mesna $(400 \mathrm{mg}$ ) was injected after IFO injection. The procedure was repeated every 3 weeks. Limb salvage surgery was conducted 2 weeks followed by two courses of neoadjuvant chemotherapy. The principle of osteosarcoma resection includes extensive resection of the capsule and at least $5 \mathrm{~cm}$ from the surgical margin to the tumor. Limb reconstruction methods included tumor inactivation and bone re-implantation, artificial knee arthroplasty, artificial prosthesis replacement and artificial hip arthroplasty. Vitro replantation was performed by ${ }^{60} \mathrm{Co}$ irradiation, and the bone cement or bone graft was used to repair the defect. Prosthetic replacement was performed with bone cement to fill the joint. Drugs and procedure of postoperative adjuvant chemotherapy were the same as neoadjuvant chemotherapy for four courses. Symptomatic treatment was given for adverse reactions in the process of chemotherapy.

The patients in group B were treated with limb salvage surgery and six cycles of adjuvant chemotherapy. Surgical methods and chemotherapy regimens were the same as those of group A.

\section{Observed indicator and evaluation criterion}

The local recurrence and metastasis rate of the two groups was observed and recorded. The severity of complications 
was classified according to the National Cancer Institute Common Toxicity Criteria (NCI-CTC v2.0). Limb function after 6 months treatment was assessed according to the Enneking limb musculoskeletal system surgical resection postoperative functional scoring system (MSTS93), including the psychological capacity, pain, muscle strength, limb stability, joint mobility and ability to live with 5 points per item and a total of 30 points. The total score $\geq 24$ is considered as excellent $(E), 18-23$ points is considered as good $(G)$, $12-17$ points is considered as feasible $(\mathrm{F})$ and $<12$ points is considered as poor $(\mathrm{P})$.

\section{Follow-up}

Upon completion of treatment, patients were evaluated by chest CT scan and orthotopic and lateral X-ray of the suffering limb, every 1 or 2 months for the first and second year, every 3 months for the third year and every 6 months thereafter. Suspected cases of metastasis or recurrent disease were confirmed by CT scan and/or bone scan.

\section{Statistical methods}

SPSS v18.0 was used for all statistical analysis. Differences between the two treatment groups were assessed using a $\chi^{2}$ test or Fisher's exact test for enumeration data and independent sample $t$-test for measurement data. Survival curves were obtained using the Kaplan-Meier method and were compared using nonparametric survival analysis (logrank test). A $P$-value $<0.05$ was considered statistically significant. Multivariate analysis was performed using the
Cox proportional hazard model with a stepwise method (forward selection), with $P$-values $<0.05$ as inclusion criteria and $P$-values $>0.10$ as exclusion criteria.

\section{Results}

\section{Patient characteristics}

All 98 patients completed the treatment in this study. Baseline characteristics showed no significant differences between group A and group B, including age, gender, tumor location, maximum tumor diameter and Enneking stage (all $P>0.05$; Table 1). Tumor bone was inactivated by Co-ray ( $70 \mathrm{~Gy}$ ) after resection and then re-implanted for 31 patients in group A, while patients in group B were not eligible for such reconstruction because they underwent surgery without neoadjuvant chemotherapy. Therefore, there was a significant difference in the type of reconstruction between the two groups $(P<0.05)$.

\section{Recurrence and metastasis}

The deadline for follow-up was December 2016. The median follow-up time was 59 months (range 36-84 months). As shown in Table 2, during the follow-up period, $14(25.0 \%)$ patients in group A and $20(47.6 \%)$ patients in group B experienced treatment failure. The metastatic site included lung and bone. In group A, five patients developed pulmonary metastases from 17 to 82 months (median 35 months) and one patient developed bone metastasis 28 months after the start of the first treatment, while in group B, six patients suffered pulmonary metastases from 13 to 64 months

Table I Comparison of general characteristics between group A and group B

\begin{tabular}{|c|c|c|c|c|}
\hline Characteristics & Group A & Group B & $\chi^{2} / t$ & P-value \\
\hline Total number of patients & 56 & 42 & - & - \\
\hline Age, ${ }^{\text {a }}$ years (range) & $23.8 \pm 3.6(12-46)$ & $22.6 \pm 3.2(13-49)$ & $1.037^{*}$ & 0.381 \\
\hline Gender (male/female) & $33 / 23$ & $24 / 18$ & 0.031 & 0.859 \\
\hline Tumor location & & & - & $0.983^{\#}$ \\
\hline Ulna & 3 & 2 & & \\
\hline Fibula & 5 & 3 & & \\
\hline Tibia & 24 & 19 & & \\
\hline Femur & 26 & 18 & & \\
\hline Maximum tumor diameter $(\mathrm{cm})$ & & & 0.817 & 0.366 \\
\hline$>10$ & 18 & 10 & & \\
\hline$\leq 10$ & 38 & 32 & & \\
\hline Enneking stage & & & 0.595 & 0.441 \\
\hline IIA & 35 & 23 & & \\
\hline IIB & 21 & 19 & & \\
\hline Type of reconstruction & & & 46.427 & 0.000 \\
\hline Tumor inactivation and bone re-implantation & 31 & 0 & & \\
\hline Artificial prosthesis replacement & 6 & 29 & & \\
\hline Artificial hip arthroplasty & 9 & 5 & & \\
\hline Artificial knee arthroplasty & 10 & 8 & & \\
\hline
\end{tabular}

Notes: aData shown as mean \pm standard deviation. ${ }^{*} t$ value; ${ }^{*}$ Fisher's exact test. 
Table 2 Comparison of the recurrence rate and metastasis rate between group $A$ and group $B$

\begin{tabular}{lll}
\hline Recurrence and metastasis & Group A (\%) & Group B (\%) \\
\hline Local recurrence & $8(14.3)$ & II $(26.2)$ \\
Metastasis & $6(10.7)$ & $8(19.0)$ \\
Local recurrence + metastasis & $0(0.0)$ & $1(2.4)$ \\
\hline
\end{tabular}

Note: $\chi^{2}=5.419, P=0.020$.

(median 22 months) and two patients developed bone metastasis 19 and 26 months after the start of the first treatment. The total metastasis and recurrence rate of group A was significantly lower than that of group B $(25.0 \%$ vs $47.6 \%$, $\chi^{2}=5.419, P=0.020$ ).

\section{Survival analysis}

At the time of this report, 13 (23.2\%) patients in group A and $18(42.8 \%)$ patients in group B died of osteosarcoma. Mortality rates of group A were significantly lower than those of group B $\left(\chi^{2}=4.282, P=0.039\right)$. Progression-free survival (PFS) and overall survival (OS) curves are shown in Figures 1 and 2, demonstrating that PFS (log-rank $\chi^{2}=4.014, P=0.045$ ) and OS (log-rank $\chi^{2}=3.859, P=0.049$ ) of group A were both significantly higher than those of group B.

\section{Adverse reactions}

There were no treatment-related deaths. Adverse reactions in both groups included hematologic toxicity, gastrointestinal

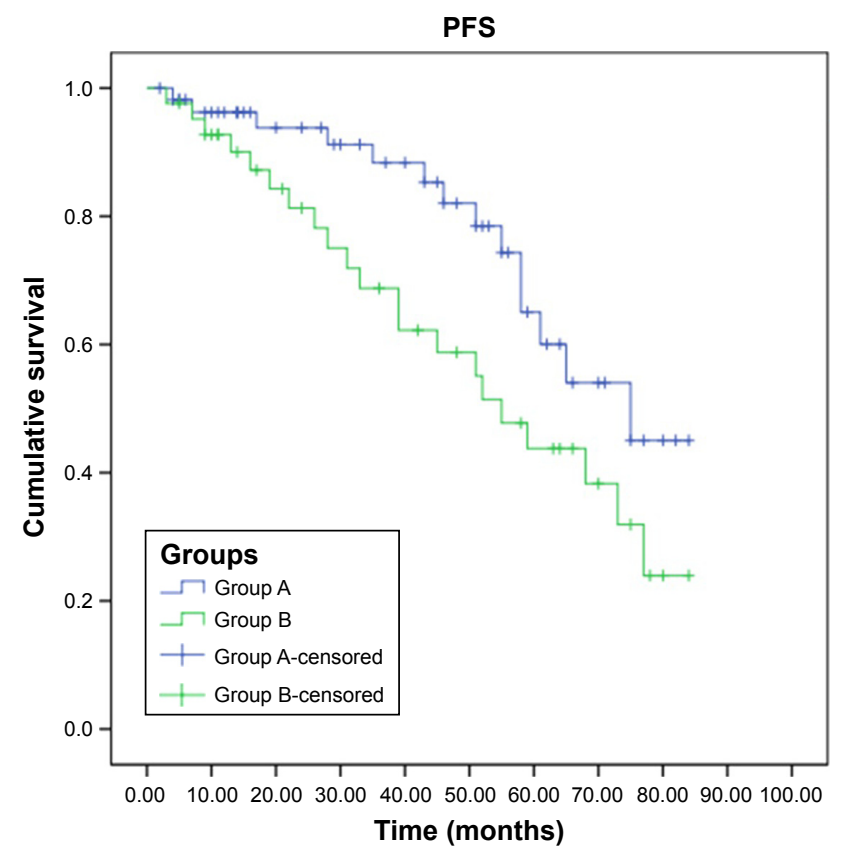

Figure I PFS for patients in group A and group B. Abbreviation: PFS, progression-free survival.

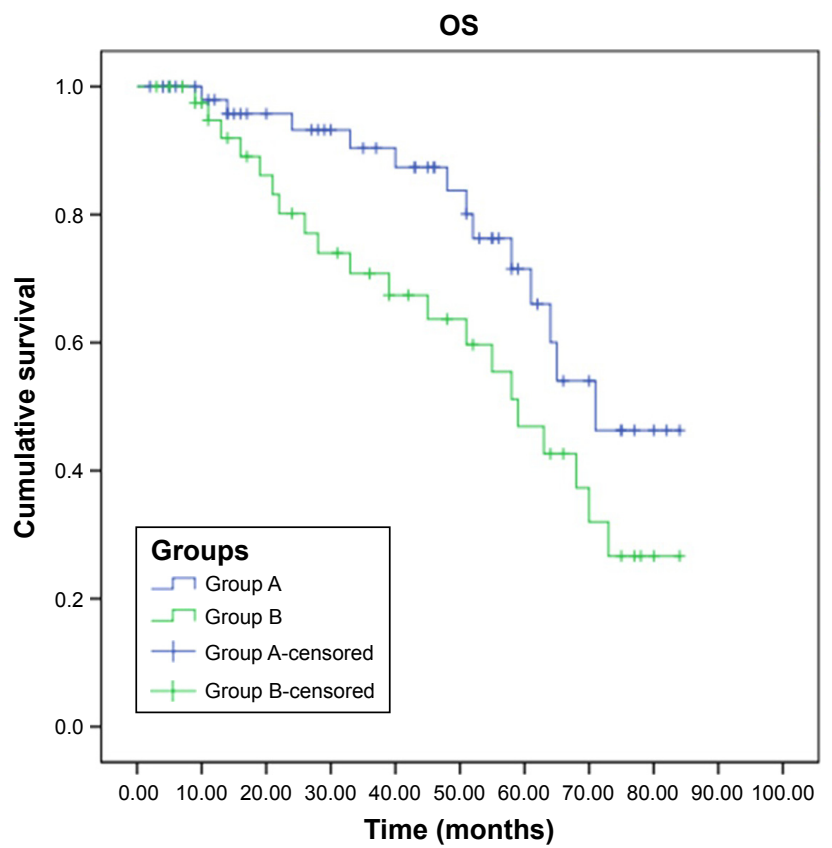

Figure 2 OS for patients in group A and group $B$. Abbreviation: OS, overall survival.

reactions and abnormal liver and kidney function, most of which were mild (grades I-II). The occurrence rate of grades III and IV adverse reaction is shown in Table 3, and there were no significant differences between group $\mathrm{A}$ and group B (all $P>0.05$ ). In addition, some patients in the two groups suffered from grades I-II peripheral neuritis, which was eased by symptomatic treatment.

\section{Limb function}

As can be seen from Table 4, the excellent and good rate of limb function in group $\mathrm{A}$ was significantly higher than that in group $\mathrm{B}$ (83.9\% vs $\left.66.7 \%, \chi^{2}=3.982, P=0.046\right)$.

\section{Discussion}

As osteosarcoma is a highly malignant systemic tumor, $80 \%$ of patients with newly diagnosed osteosarcoma have had blood micrometastases. ${ }^{10}$ Most patients died of distant metastases, mainly lung metastases after surgery. In the past,

Table 3 Comparison of the occurrence of grades III and IV adverse reactions during chemotherapy of the two groups

\begin{tabular}{lllll}
\hline Adverse reactions & Group A & Group B & $\chi^{2}$ & P-value \\
\hline Hematologic toxicities & 13 & 9 & 0.044 & 0.834 \\
Leukopenia & 8 & 3 & & \\
Thrombocytopenia & 5 & 6 & & \\
Gastrointestinal tract reaction & 5 & 4 & - & $1.000^{\#}$ \\
Hepatic and renal dysfunction & 2 & I & - & $1.000^{\#}$ \\
\hline
\end{tabular}

Note: "Fisher's exact test. 
Table 4 Comparison of the limb function between group $A$ and group $B$

\begin{tabular}{lll}
\hline Limb function & Group A (\%) & Group B (\%) \\
\hline $\mathrm{E}$ & $27(48.2)$ & $15(35.7)$ \\
$\mathrm{G}$ & $20(35.7)$ & $13(31.0)$ \\
$\mathrm{F}$ & $7(12.5)$ & $7(16.7)$ \\
$\mathrm{P}$ & $2(3.6)$ & $7(16.7)$ \\
$\mathrm{E}$ and G & $47(83.9)$ & $28(66.7)$ \\
\hline
\end{tabular}

Note: $\chi^{2}=3.982, P=0.046$

Abbreviations: E, excellent; G, good; F, feasible; P, poor.

patients with limb osteosarcoma were mainly treated with amputation, which would lead to loss of limb function and reduced the quality of life of the patients. In the treatment of patients with limb osteosarcoma, physicians and patients not only wish to extend the survival time of patients but also aim to retain the diseased limb and save its function, and thereby improve patients' quality of life. With the advent of bone graft with blood vessels, artificial prosthesis replacement and resection and inactivation and replantation of tumor bone, amputation surgery has been gradually replaced by limb salvage surgery, which has now become the standard procedure for limb osteosarcoma. ${ }^{11,12}$

Due to the combination of aggressive chemotherapy and surgery, the long-term survival for patients with osteosarcoma increased from $10 \%-15 \%$ to $65 \%-70 \%{ }^{13}$ In 1982 , Rosen et $\mathrm{al}^{14}$ first proposed preoperative chemotherapy for the treatment of limb osteosarcoma. Neoadjuvant chemotherapy has many advantages. First of all, this method can limit or shrink the primary lesion, eliminate tumor satellite lesions and increase opportunities of limb surgery or reduce the difficulty of surgery. Neoadjuvant chemotherapy can eliminate the micrometastases in the blood and reduce tumor recurrence rate. In addition, effectiveness of chemotherapy drugs can be assessed according to tumor cell necrosis rate, and based on this, postoperative chemotherapy drugs would be prescribed. ${ }^{15}$ It is reported that neoadjuvant chemotherapy has greatly increased the 5 -year survival rate of patients with osteosarcoma. ${ }^{16,17}$

The EPI + DDP + MTX + IFO regimen used in this study is an internationally recognized first-line regimen for the treatment of osteosarcoma. ${ }^{18,19}$ EPI, DDP and IFO are all cell cycle non-specific drugs. EPI can directly embed in DNA base pairs, split DNA bonds and interfere in the synthesis of DNA and RNA; at the same time, it can damage the structure and function of cell membranes..$^{20} \mathrm{DDP}$ inhibits DNA replication and cancer cell division by binding to DNA and forming a platinum compound. IFO kills tumor cells by cross-linking with DNA. EPI, DDP and IFO have synergistic effects and can significantly reduce the occurrence of drug resistance. ${ }^{21}$ MTX, as a kind of cell cycle-specific drug, acts on the $\mathrm{S}$ phase of the cell cycle and affects the biosynthesis of the purine nucleotides and pyrimidine nucleotides in tumor tissue by inhibiting the dihydrofolate reductase, and thereby achieves the effect of inhibiting tumor cell proliferation. Kudawara et $\mathrm{al}^{8}$ have used this regimen for the treatment of non-metastatic osteosarcoma of the extremities and demonstrated the 5-year event-free and overall survival rates to be $83 \%$ and $98 \%$. In this study, the tumor cell necrosis rates in group A assessed during operation were all $>90 \%$, which further illustrated that osteosarcoma cells were sensitive to these chemotherapy drugs. Therefore, the EPI + DDP + $\mathrm{MTX}+\mathrm{IFO}$ regimen has continued to be used for postoperative chemotherapy.

In 2009, we introduced neoadjuvant chemotherapy combined with limb salvage surgery to treat some patients with limb osteosarcoma. This study retrospectively analyzed the patients with osteosarcoma treated in our hospital from January 2010 to December 2013. The total metastasis and recurrence rate of group A was significantly lower than that of group B, and the Kaplan-Meier method showed that PFS and OS of group A were both significantly higher than those of group B, indicating that neoadjuvant chemotherapy can give patients with osteosarcoma more survival benefit. The excellent and good rate of limb function in group A was significantly higher than that in group B, illustrating that neoadjuvant chemotherapy can reduce the tumor size, help to find the best surgical resection margin, retain limb function with the greatest degree and achieve the purpose of local radical therapy. Preventive medication or symptomatic support was given to all patients during chemotherapy, and all patients tolerated the chemotherapy well. In addition, there was no significant difference in the incidence of grades III-IV adverse reactions between the two groups.

\section{Conclusion}

This study demonstrated the excellent outcome of neoadjuvant chemotherapy combined with limb salvage surgery for the patients with limb osteosarcoma of Enneking stage II. However, this study is a retrospective study with a small sample size. In the future, a multicenter, randomized controlled trial with larger sample size will be conducted to achieve more reliable results.

\section{Acknowledgment}

We would like to express our sincere gratitude to all patients who participated in this study. 


\section{Disclosure}

The authors report no conflicts of interest in this work.

\section{References}

1. Salah Z, Arafeh R, Maximov V, et al. miR-27a and miR-27a contribute to metastatic properties of osteosarcoma cells. Oncotarget. 2015;6(7):4920-4935.

2. Isakoff MS, Bielack SS, Meltzer P, Gorlick R. Osteosarcoma: current treatment and a collaborative pathway to success. J Clin Oncol. 2015; 33(27):3029.

3. Anderson P. Liposomal muramyl tripeptide phosphatidyl ethanolamine: ifosfamide-containing chemotherapy in osteosarcoma. Future Oncol. 2015;2(3):333-343.

4. Luetke A, Meyers PA, Lewis I, et al. Osteosarcoma treatment - where do we stand? A state of the art review. Cancer Treat Rev. 2014;40(4): $523-532$.

5. Hegyi M, Semsei AF, Jakab Z, et al. Good prognosis of localized osteosarcoma in young patients treated with limb-salvage surgery and chemotherapy. Pediatr Blood Cancer. 2012;58(4):415.

6. Zhang HJ, Yang JJ, Lu JP, et al. Use of intra-arterial chemotherapy and embolization before limb salvage surgery for osteosarcoma of the lower extremity. Cardiovasc Intervent Radiol. 2009;32(4):672-678.

7. Gaetano Bacci MD, Alessandra LMD, Michela VMA, et al. Prognostic factors for osteosarcoma of the extremity treated with neoadjuvant chemotherapy. Cancer. 2006;106(5):1154-1161.

8. Kudawara I, Aoki Y, Ueda T, et al. Neoadjuvant and adjuvant chemotherapy with high-dose ifosfamide, doxorubicin, cisplatin and high-dose methotrexate in non-metastatic osteosarcoma of the extremities: a phase II trial in Japan. J Chemother. 2013;25(1):41.

9. Bacci G, Ruggieri P, Bertoni F, et al. Local and systemic control for osteosarcoma of the extremity treated with neoadjuvant chemotherapy and limb salvage surgery: the Rizzoli experience. Oncol Rep. 2000;7(5):1129.

10. Whelan JS, Bielack SS, Marina N, et al; EURAMOS Collaborators. EURAMOS-1, an international randomised study for osteosarcoma: results from pre-randomisation treatment. Ann Oncol. 2015;26(2): 407-414.

11. Qadir I, Umer M, Baloch N. Functional outcome of limb salvage surgery with mega-endoprosthetic reconstruction for bone tumors. Arch Orthop Trauma Surg. 2012;132(9):1227-1232.
12. Kayalar M, Sabah D, Yuceturk G. Peroperative flap monitorization with fluorescein in limb salvage operations. Educ Theatre J. 1976;28(4): 445-448.

13. Ferrari S, Serra M. An update on chemotherapy for osteosarcoma. Expert Opin Pharmacother. 2015;16(18):2727.

14. Rosen G, Caparros B, Huvos AG, et al. Preoperative chemotherapy for osteogenic sarcoma: selection of postoperative adjuvant chemotherapy based on the response of the primary tumor to preoperative chemotherapy. Cancer. 1982;49(6):1221-1230.

15. Bacci G, Ferrari S, Longhi A, et al. Neoadjuvant chemotherapy for high grade osteosarcoma of the extremities: long-term results for patients treated according to the Rizzoli IOR/OS-3b Protocol. J Chemother. 2013;13(1):93-99.

16. Ferrari S, Ruggieri P, Cefalo G, et al. Neoadjuvant chemotherapy with methotrexate, cisplatin, and doxorubicin with or without ifosfamide in nonmetastatic osteosarcoma of the extremity: an Italian sarcoma group trial ISG/OS-1. J Clin Oncol. 2012;30(17):2112-2118.

17. Collins M, Wilhelm M, Conyers R, et al. Benefits and adverse events in younger versus older patients receiving neoadjuvant chemotherapy for osteosarcoma: findings from a meta-analysis. J Clin Oncol. 2013; 31(18):2303.

18. Niu BH, Wang JJ. Establishment of adriamycin resistance cell lines of human osteosarcoma and their biological characteristics. J Fourth Mil Med Univ. 2009;30(1):41-44.

19. Flarakos CC, Weiskopf A, Robinson M, et al. Metabolism of selective 20-Epi-vitamin D3, analogs in rat osteosarcoma UMR-106 cells: isolation and identification of four novel C-1 fatty acid esters of $1 \alpha, 25$ dihydroxy-16-ene-20-Epi-vitamin D3. Steroids. 2017;119:18-30.

20. Roché H, Fumoleau P, Spielmann M, et al. Sequential adjuvant epirubicin-based and docetaxel chemotherapy for node-positive breast cancer patients: the FNCLCC PACS 01 trial. J Clin Oncol. 2006; 24(36):5664.

21. Brocato N, Bruno MF, Araujo CE, et al. Treatment of non-small cell lung cancer with ifosfamide (IFO) + 4'-epiadriamycin (EPI) + platinum vs. IFO + EPI: a GETLAC Study. Oncology. 1995;52(1):24-31.
OncoTargets and Therapy

\section{Publish your work in this journal}

OncoTargets and Therapy is an international, peer-reviewed, open access journal focusing on the pathological basis of all cancers, potential targets for therapy and treatment protocols employed to improve the management of cancer patients. The journal also focuses on the impact of management programs and new therapeutic agents and protocols on

\section{Dovepress}

patient perspectives such as quality of life, adherence and satisfaction. The manuscript management system is completely online and includes a very quick and fair peer-review system, which is all easy to use. Visit http://www.dovepress.com/testimonials.php to read real quotes from published authors. 